\section{Fullerenes Add Motion to Micromachines}

In order to make machines on the molecular scale, researchers need to be able to fabricate components that are analogous to those of macromachines, such as gears, wheels, pistons, valves, and bearings. Fullerenes, with their sphere-like structure, would seem to be ideal for use as bearings, but early work involving these $\mathrm{C}_{60}$ molecules between two surfaces was less than encouraging. Multilayers of $C_{60}$ between plates deformed elastically, leading to high friction. However, results reported in the February 7 issue of Physical Review Letters suggest that monolayers of $\mathrm{C}_{60}$ sandwiched between silicon surfaces coated with graphite may produce a nearly frictionless bearing system for use in micromachines.

While graphite is a well-known lubricant, its function in this bearing system is not one of lubrication. Rather, the hexagonal carbon faces on the top and bottom of the $\mathrm{C}_{60}$ molecule "mesh" with the sixmember carbon rings that form the sheets of the graphite sandwich to form nanogears; these nanogears allow the $C_{60}$ molecules to roll between the graphite layers, acting as bearings. The action is similar to rolling a ball between the hands by moving them in parallel but opposite directions.

K. Miura and S. Kamiya of Aichi University of Education in Kariya, Japan, along with N. Sasaki of Sekei University in Tokyo, used frictional force-mapping techniques to show that $\mathrm{C}_{60}$ forms a close-packed monolayer on graphite. Furthermore, the monolayer can be formed initially with hexagonal faces of $\mathrm{C}_{60}$ maintaining $\mathrm{AB}$ stacking with the hexagonal carbon rings of the graphite on both sides of the sandwich.

When a small torque is applied to the system, the $\mathrm{C}_{60}$ molecules roll, ideally in a direction that will find their hexagonal faces once again aligned with the hexagonal rings of the graphite sheets on either side. However, because the $\mathrm{C}_{60}$ molecule is not composed exclusively of hexagonal faces but includes pentagonal faces as well, there is no guarantee that this ideal alignment will occur each time. If a pentagonal face of $\mathrm{C}_{60}$ comes into contact with the graphite hexagon, the gear will briefly stick until thermal energy causes rotation of the $\mathrm{C}_{60}$ so that a hexagonal face once again matches up with the graphite sheet. From this position, the $C_{60}$ bearing can then turn again. The bearing system thus moves by a stick-slip mechanism at room temperature. Because the energy required to overcome the sticking is relatively low, the researchers believe that at slightly elevated temperatures, the bearings will roll smoothly.

This first demonstration of a practical molecular bearing system, coming as it does with a fundamental understanding of the mechanism involved, could help add the long-awaited capability of movement to previously static micromachines.

"Silicon substrates covered with graphite should be useful as movable parts of nano- and micromachines," said Miura. "This system is expected to be useful in MEMS [microelectromechanical systems] devices."

Jean Michel Martin, head of the Department of Materials and Surface Science at the Ecole Centrale de Lyon in France, while finding these results important, expressed concern that the experiments were not conducted in a high vacuum environment.

"The presence of water molecules and even oxygen molecules are well known to interact with $\mathrm{C}_{60}$ and graphite flakes," he said. If present, these molecules could

\section{GLASSMAN HIGH VOLTAGE DC Power Supply Solutions for Vacuum Processing Applications For small DC Sputtering sources, DC bias, lon sources and Glow Discharge Plasma Requirements}

For over 25 years the Glassman name is synonymous with High Reliability Performance and has built it's reputation as a market leader by it's dedication and investment in technology development as well as after-sale customer support that is second to none in the power supply industry.

For Vacuum processing applications, Glassman's $\mathrm{FX}(300 \mathrm{~W}), \mathrm{EK}(600 \mathrm{~W})$ and $\mathrm{EQ}(\mathrm{I} .2 \mathrm{~kW})$ series power supplies will meet your needs for low-mid power requirements, with typical application output voltage range from $300 \mathrm{~V}-10 \mathrm{kV}$. All feature compact size, advanced arc management, low stored energy, and use air as the primary dielectric-A Glassman Innovation! Key Features:

- Proprietary Arc Sense and Arc Quench circuitry for ultimate power supply and load protection

- High frequency technology-up to $60 \mathrm{kHz}$

- Tight regulation and high stability

- Low stored output energy

- RS-232 serial interface option

For more information on the complete line of Glassman High Voltage power supplies, visit our website at www.glassmanhv.com or call us today at $908-638-3800$.
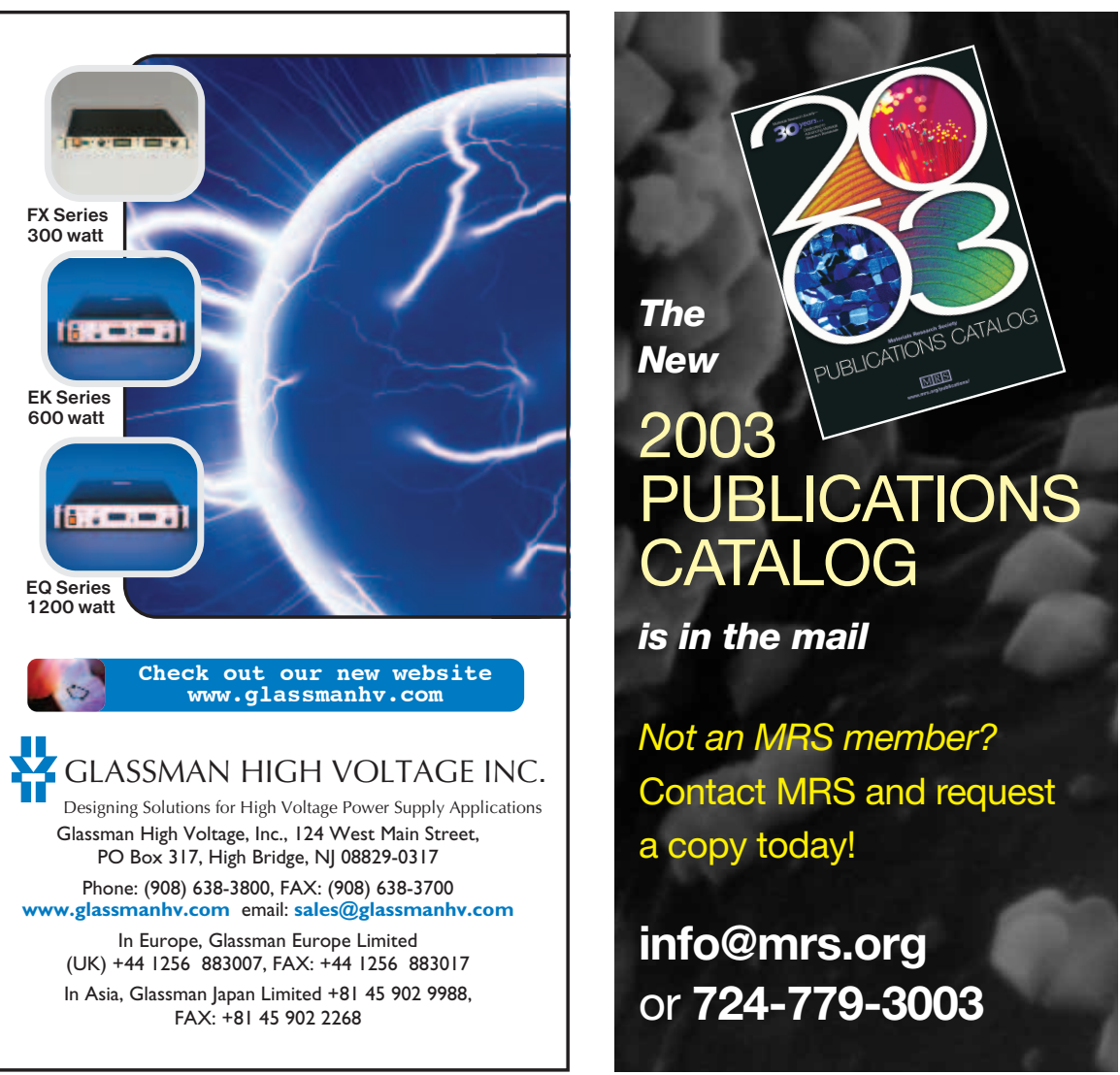
be playing a role in the proposed stickslip mechanism.

Tim PALUCKA

\section{Two-Photon Polymerization of Organically Modified Ceramics Used in 3D Microfabrication}

Two-photon polymerization (2PP) of photosensitive acrylates and epoxies has been used in recent years to fabricate threedimensional (3D) microstructures such as photonic crystals and mechanical devices. Femtosecond laser pulses, which initiate the $2 \mathrm{PP}$, can achieve submicrometer resolution when tightly focused into the volume of liquid resin. B.N. Chichkov of Laser Zentrum Hannover, R. Houbertz of the Fraunhofer-Institut für Silicatforschung in Würzburg, and co-workers have extended this technique to a commercial multifunctional inorganic-organic hybrid polymer that has properties advantageous for photonic applications. They have applied this technique to the fabrication of microstructures and photonic crystals with a structure size down to $200 \mathrm{~nm}$ and a periodicity of $450 \mathrm{~nm}$.

As reported in the March 1 issue of Optics Letters, the material employed for 2PP was ORMOCER, an acronym for organically modified ceramic, developed at the Fraunhofer-Institut für Silicatforschung. The researchers recognized that ORMOCERs, which are produced by a solgel method, have many properties important for photonics, such as an adjustable index of refraction in the range of 1.47-1.56, high optical transparency with low losses for both the resin and polymerized material, high chemical resistance, and exceptional thermal and mechanical properties. Specifically, ORMOCER-1 is designed for UV photolithography and contains the UVsensititve Irgacure 369 initiator. All ORMOCERs are transparent in the IR and especially at $780 \mathrm{~nm}$, so that Ti-sapphire laser pulses can be focused into the volume of liquid resin.

The research team used a Ti-sapphire oscillator with a repetition rate of $80 \mathrm{MHz}$, a pulse duration of $80 \mathrm{fs}$, and a laser wavelength of $780 \mathrm{~nm}$. A mechanical shutter with a minimum switching time of $5 \mathrm{~ms}$ controlled the number of laser pulses and the radiation time. Femtosecond laser pulses were focused with a $100 \times$ oilimmersion-lens microscope objective with a numerical aperture of 1.4 and $n_{\text {oil }}=1.515$ for index matching. The researchers said that resolution beyond the diffraction limit can be achieved because of the threshold behavior of the $2 \mathrm{PP}$ process.

The researchers developed expressions for the diameter and length of the polymerized volume (volume pixel, or voxel) as functions of laser power and irradiation time. By curve-fitting the experimental data to these expressions, the researchers were able to determine the expressions' unknown parameters, which in turn define the lateral and axial resolution of the objective ( $340 \mathrm{~nm}$ and $1.2 \mu \mathrm{m}$, respectively).

The researchers demonstrated 3D microstructuring with femtosecond lasers and fabrication by $2 \mathrm{PP}$ by presenting scanning electron micrographs of a micrometer-scale statue of the Venus de Milo. The researchers scanned the laser beam with an $x-y$ galvo scanner and moved the sample in the $z$ direction with a translation stage. In order to speed fabrica- tion, only the shell of the statue was irradiated with femtosecond laser pulses. After washing out the remaining liquid resin, the statue was irradiated with UV light for final polymerization of the inner body. Total fabrication time was about $5 \mathrm{~min}$. The researchers also fabricated microcapsules from ORMOCER-1, which is biocompatible and can be used in medical applications. A third demonstration was the fabrication of a photonic crystal, which was built up from individual rods with diameters of $200 \mathrm{~nm}$ and an inter-rod spacing of $250 \mathrm{~nm}$. Total fabrication time was about $10 \mathrm{~min}$.

The researchers said they expect that

\section{Cost-Effective Portable Spin Coater}

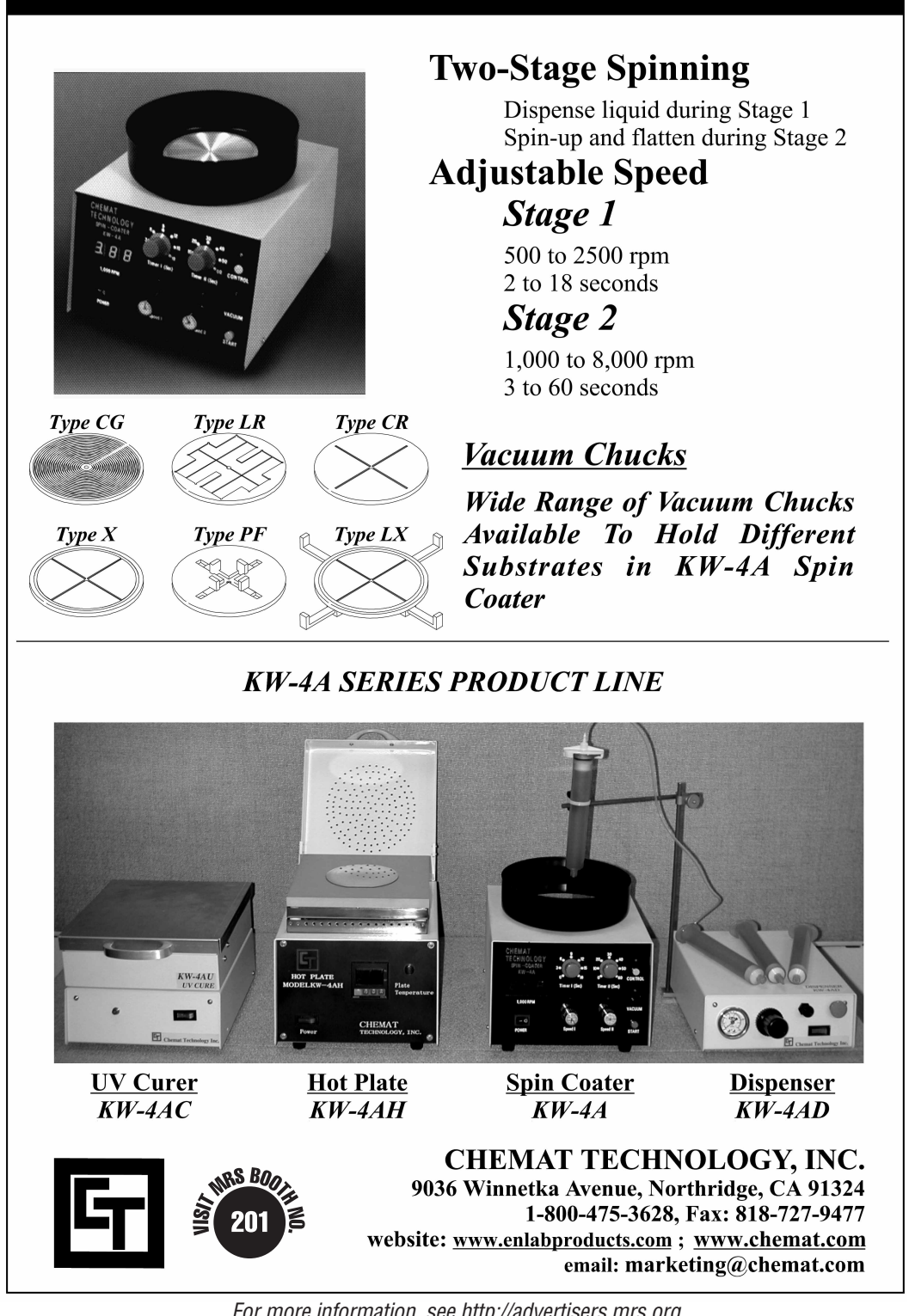

For more information, see http://advertisers.mrs.org 